Running head: Physicality of mind

\title{
Effects of stimulus processing on event-related brain potentials of close others
}

\author{
Maud Haffar ${ }^{1}$, Hugo Pantecouteau ${ }^{2}$, Sheila Bouten $^{3} \&$ J. Bruno Debruille ${ }^{1,3,4, *}$
}

${ }^{1}$ Department of Neuroscience, McGill University, Montréal, Canada

${ }^{2}$ École Normale Supérieure de Lyon, Lyon, France

${ }^{3}$ Douglas Mental Health University Institute, Montréal, Canada

${ }^{4}$ Department of Psychiatry, McGill University, Montréal, Canada

\section{*Corresponding Author:}

Dr. J. Bruno Debruille,

Cognitive and Social Neurosciences Lab,

Douglas Hospital Research Center,

6875 boulevard LaSalle

Montréal (QC), H4H 1R3

Canada

Email: bruno.debruille@,mcgill.ca

Keywords: qualia, consciousness, emission theories, perception, event-related brain potentials, P600 or late posterior positivity, N400. 
Abstract We take what we see, hear, smell and feel for the reality. However, as neuroscientists, we know that this reality, that is, our perceptual world, is in fact made up by the brain from the processing of the nerve impulses coming from receptors. Ancient Greeks used to think that this perceptual world, sometimes called our 3D movie (Chalmers), is emitted and has its own physical nature. Given how real the 3D movie looks to us, it is still difficult today to consider that all we would be dealing with would be patterns of brain activity The present study thus aimed at testing whether the perceptual world could have some physical existence in addition to that of the neural patterns responsible for it. To achieve that goal, we tried to see whether brains could be sensitive to the 3D movie of others. This, admittedly unusual, operational hypothesis was based on two assumptions. First, brains are sensitive to the $3 \mathrm{D}$ movie, as our experience includes reactions to our perceptual world. Second, the physicality at stake does not differ across individuals. We recorded the event-related brain potentials (ERPs) evoked by stimuli of the international affective picture system in pairs of closely-related participants. Most importantly, they could neither see the stimuli simultaneously presented to their partners nor their reactions to them. As in Bouten et al. (2015), around $400 \mathrm{~ms}$ after the onset of the stimuli, ERPs started being more positive in inconsistent conditions. Namely, when the two subjects of each pair were presented with the same stimulus whereas they were told it would be a different one and vice-versa (i.e., different-stimuli expected to be same). ERPs were less positive when the two subjects of a pair were presented with the same stimuli and were told they were the same and conversely (i.e., different-stimuli expected to be different). The same experiment was then run in pairs of strangers. No significant effect of consistency on ERPs was observed even though participants could, this time, see, in the very periphery of their visual field, the reactions of their partner to the stimuli. We thus use the results of both studies to support a new version of the emission theory of consciousness and to suggest that the sensitivity to the perceptual world of others may depend on their prior familiarity with it. 


\section{Introduction}

Cognitive neuroscience promotes the idea that each aspect of consciousness corresponds to a particular pattern of neural activities. However, it is hard to imagine that colors and sounds are such patterns. What is it in sets of neuronal discharges that could be a color or a smell? Moreover, many patterns of neural activities are known to be deprived of any conscious correlate (Bodart et al., 2017; Silverstein et al. 2015; Huang et al., 2016) and no obvious reason has been found as to why some other patterns would have such correlates. Complexity has been proposed as an answer to this question (e.g., Tononi \& Edelman, 1998), but in and of itself, it is difficult to understand why a quale, such as red, would emerge when neural activity patterns become more complex or when the activities of several cortical areas become related to each other (as in Dehaene et al. 2014).

Finally, many authors refer to conscious information processing (e.g., Dehaene et al. 2016; Tononi et al., 2016). However, conscious experience appears to pertain to outputs rather than to processes themselves: when we open our eyes on a new picture, the way it appears to us is devoid of the brain computations that have enable us to see it.

Ancient Greek philosophers, such as Empedocles, as well as, Plato, Pythagoras, Ptolemy and Galen, faced the existence of that perceptual world, called our 3D movie by Chalmers (2014). Today, they might still propose that something (other than light, this time) is emitted by the subject. Often ridiculed (e.g. see “Ancient Visions," n.d.), their purportedly obsolete emission theory could be revamped. Bioelectrical activities of neurons triggered by external stimuli could output phenomena, the characteristics of which would be specific to each quale but whose physics would remain to be found.

The goal of the present paper is to test whether brain activities responsible for consciousness, and more particularly vision, could engender a physical phenomenon and see whether qualia are underlain by both these activities and that phenomenon. In other words, we are 
examining here whether the perceptual world generated by neural activities really exists and thus has its own physical nature. Like the tungsten filament of a bulb heated by the electrical current emits a phenomenon of a different physical nature: light; we are hypothesizing that some types of bioelectrical activity of neurons can emit a phenomenon of a different physical nature, of which our $3 \mathrm{D}$ movie is made of.

Fortunately, this unusual hypothesis leads to a straightforward prediction: Had the perceptual world a physical nature of its own, it would then have to have some physical impacts. Moreover, the first of these impacts should be on brains themselves, for two reasons. First because we are sensitive to our percepts. We are sometimes even surprised by them despite the fact that they are produced by our brain itself. Second, because, if we were not sensitive to the 3D movie, brains would have no means to regulate its production. This would contrast with other productions, such as that of motor behaviors or hormones and neurotransmitters, which are all regulated through the effects they have on brains themselves, via proprioception for motor productions, via effects of hormones on the enzymes that catalyze their production and via effect of neurotransmitters on their pre-synaptic receptors.

However, at first sight, this prediction of an effect of the perceptual world on the brain that produces it does not appear falsifiable. There seems to be no way to tease apart indexes of this effect from indexes of the production of such world by bioelectrical activities of brain cells. Nevertheless, the physical nature of perceptual worlds should not be different across individuals. Thus, if the perceptual world of a subject can impact the activity of the brain that produced it, it might also impact the activity of other brains. And, if this, apparently unlikely, latter impact were to be detected, it would directly prove that perceptual worlds are emitted and that their physics is not limited to bioelectrical activities of neurons within the brain. 
In an attempt to search for such an impact of perception on others, Bouten et al.'s (2015) recorded the electroencephalogram (EEG) of persons who were sitting next to a subject to whom stimuli were presented. These persons could not see the stimuli presented to the subject, as the aim was to see whether the perceptual stimulus created by the subject in response to this presentation had an impact on the activity of the brain of that other person. In order to increase the number of participants tested, the experimental design was reciprocal, that is, the other person was simultaneously presented with stimuli and the subject's EEG was also recorded. Again, in order to focus on the hypothesized effect of the perceptual stimulus of each subject on the brain activity of his/her partner, the two participants of each pair were not able to see the real stimulus presented to their partner.

To detect the hypothesized impact, the sameness of the stimuli presented was manipulated. In two blocks of trials, the "same-blocks", both participants of each pair saw the same stimuli, and, in two other blocks of trials, the "different-blocks", they saw different ones. This manipulation was based on the idea that if the brain of the participant is sensitive to the percepts created by his/her partner in response to the stimulus, these reactions might then depend on the nature of that stimulus. To detect such reaction differences, the recorded EEGs were used to compute the event-related brain potentials (ERPs) elicited by the stimuli in the two block-conditions.

On the other hand, the expectations of the participants as to this stimulus-sameness were manipulated in order to see whether the hypothesized effect of sameness on ERPs could depend on them. Thus, in one of the two "same-blocks" and in one of the two "different-blocks", subjects were told that they were going to be presented with the same stimuli. In the other of the two same-blocks and in the other of the two different-bocks, they were told that they would see different ones. 
A simple 2 x 2 design was thus used (i.e., 2 stimulus sameness $\times 2$ sameness-expectation conditions). Any ERP modulation by the actual sameness of the stimuli was planned to be used as a support of the sensitivity of others to the perceptual stimulus of the subject.

The ERPs elicited by the visual stimuli used (i.e., pictures of the international affective picture system, the IAPS, Lang et al., 1997) were found to depend on the combination of the two variables manipulated despite the impossibility for participants to see the actual stimulus their partner was presented with. About $400 \mathrm{~ms}$ after the onset of the two stimuli, ERP voltages started to be more positive when the expectation was inconsistent with reality than when it was consistent (Fig. 1). In other terms, ERPs were more positive for the "different-stimuli expected to be the same" and the "same-stimuli expected to be different" conditions than for the "same-stimuli expected to be the same" and the "different-stimuli expected to be different" conditions. The 32 subjects of the 16 pairs tested were then split into 2 subgroups to see whether this consistency-difference could be found in each of the two subgroups. This was the case. On the other hand, debriefing sessions revealed that participants did not detect any deception. Thus, only a sensitivity to the hypothesized physical emissions of perceptual stimuli could be responsible for the ERP differences found.

Nevertheless, before making such a strong conclusion, every possible bias has to be considered. First, during the experiment, the two participants could see each other in the very periphery of their visual fields. Given that some of the IAPS stimuli used were shocking, they might have triggered postural and facial reactions. These reactions could have been automatically processed by the persons and might have led to an unconscious detection of the falsity of the statements aimed at generating the expectations. Such hypothetical covert detections could have then impacted the way stimuli of the whole block were processed and thus, be responsible for the ERP differences found between block-conditions. There was thus a need to see whether the effect 
of consistency on ERPs could be observed when the two participants of a pair could not see each other.
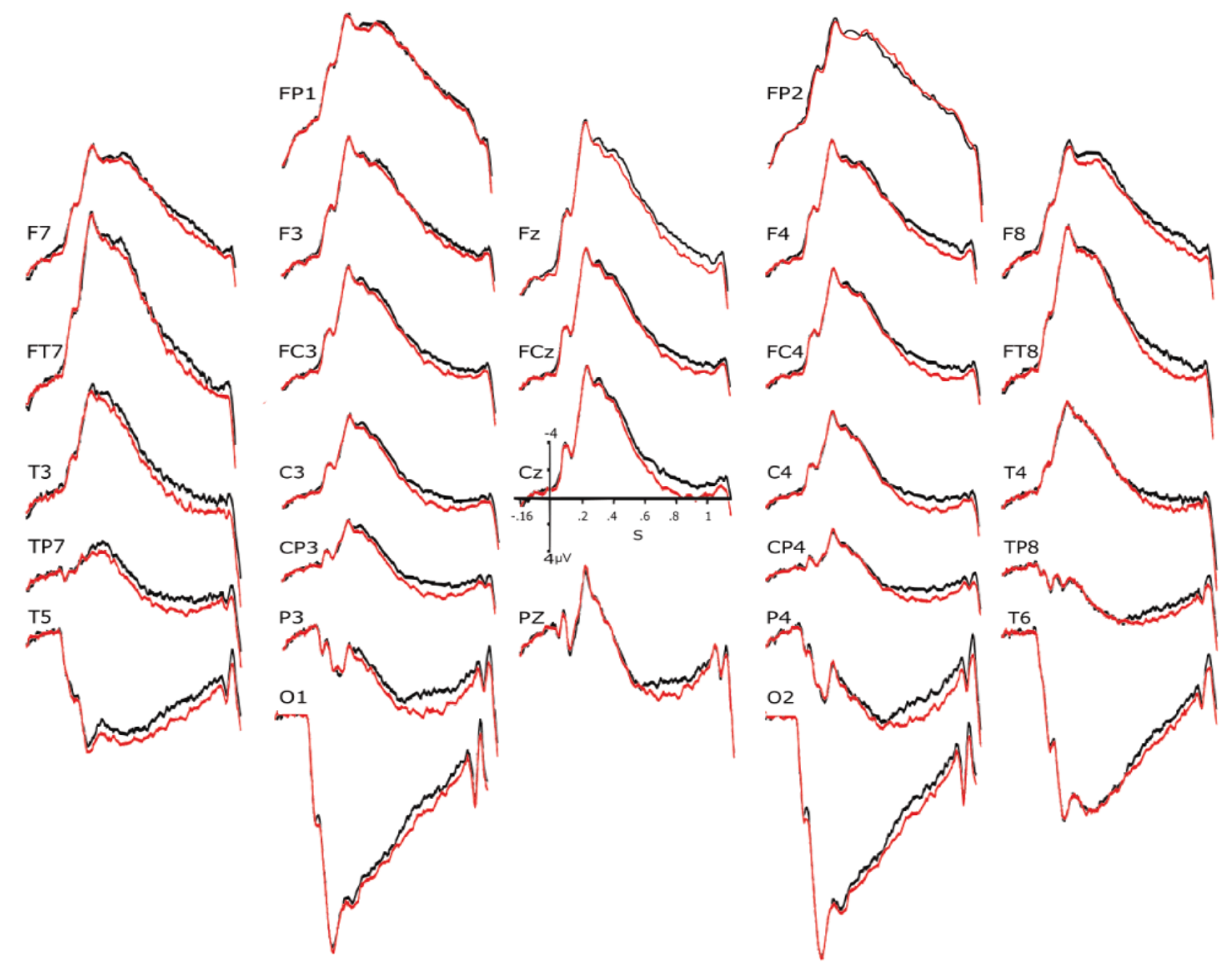

Figure 1. Grand $(n=32)$ averages of the ERPs evoked by IAPS pictures in the Bouten et al.'s (2015) study. The voltage is represented on the y-axis in microvolts with negative values upward. Time is represented on the $\mathrm{x}$-axis in seconds. The onset of the stimulus is at $0 \mathrm{~s}$. Black lines correspond to the two gathered blocks where reality and expectation were consistent with each other (see Introduction section). Red lines correspond to the two gathered blocks where reality and expectation were inconsistent. 
On the other hand, pairs of participants were chosen among close friends, siblings or couples, as it was thought that partners more used to each other could be more sensitive to, and/or more capable of being sensitive to the perceptual stimulus of their partner. It is thus unknown whether the consistency effect could be found with pairs of strangers.

The first experiment of the present study was aimed at addressing the first issue. The goal of the second experiment was to focus on the latter one. 
Experiment 1: Testing whether Bouten et al. (2015) results can be found when closely related people are visually isolated from each other

\section{Introduction}

As mentioned, in Bouten et al. (2015), some (i.e., about 15\%) of the IAPS images used as stimuli were quite emotionally laden. Therefore, they might have triggered subtle body movements or postural reactions from participants. The partner could have processed these reactions given that the two subjects of each pair were not visually isolated. Despite the absence of conscious detection of stimulus inconsistency revealed by the debriefing session, there could have been a covert detection, which could have generated the consistency effect on ERPs reported in Bouten et al. (2015). The first experiment of the present study was thus done to see if their results could be replicated when subjects cannot see each other. A curtain was thus placed between them, in addition to the screen's cardboard, which prevented them from seeing the stimuli presented to their partner. New pairs of closely related people were recruited and the same procedure as that of Bouten et al. (2015) was used.

\section{Methods}

Participants

Thirty-two right-handed, English speaking participants $(23 \mathrm{~F}, 9 \mathrm{M}$; mean age $=21.1$, SD $=1.9)$ from couples, pairs of friends or siblings were recruited. They all had a university degree or were in the process of completing it. An eligibility questionnaire was administered to ensure that participants had known each other for at least three years and to exclude participants abusing drugs and/or alcohol. The other exclusion criteria were a history of psychiatric disorder, taking a 
medication related to such a disorder or a history of schizophrenia or bipolar disorder in one of their first-degree relatives.

Consent

Each participant read and signed an informed consent form accepted by the Douglas Institute Research and Ethics Board. This board, which follows the principles expressed in the declaration of Helsinki, approved the design of the study (Douglas REB \#12/12).

\section{Stimuli and procedure}

Stimuli were 280 pictures of the international affective picture system (IAPS). Each of these pictures were randomly attributed to one of four 70-picture sets: A, B, C and D. Among each set, several striking pictures were kept to maintain participants' attention during the task. These 4 sets were used to create the four sameness-expectation block-conditions by building 2 ensembles of double pictures, one picture for each of the two subjects of a pair at each trial, AA, BB, CD, DC and AB, BA, CC, DD. Half of the pairs of participants had the $1^{\text {st }}$ ensemble while the other half had the second. Each of the 280 pictures thus appeared as often in the each of the four samenessexpectation block-conditions across pairs of participants. The order of presentation of the blockconditions was randomized across subject pairs using a Latin square so that no effect of fatigue could be responsible for ERP difference between these conditions. The two subjects of each pair sat next to each other in front of the computer screen. A vertical piece of cardboard perpendicular to the screen prevented them from seeing their partner's part of the screen so they could not see if they were presented with the same stimulus as their partner or not. They were also separated by a curtain 
Running head: Physicality of mind

so that they could not see each other. The distance from their eyes to the screen was $60 \mathrm{~cm}$ and the vision angle encompassed by each stimulus was $9^{\circ}$, as illustrated in Fig. 2A (below).
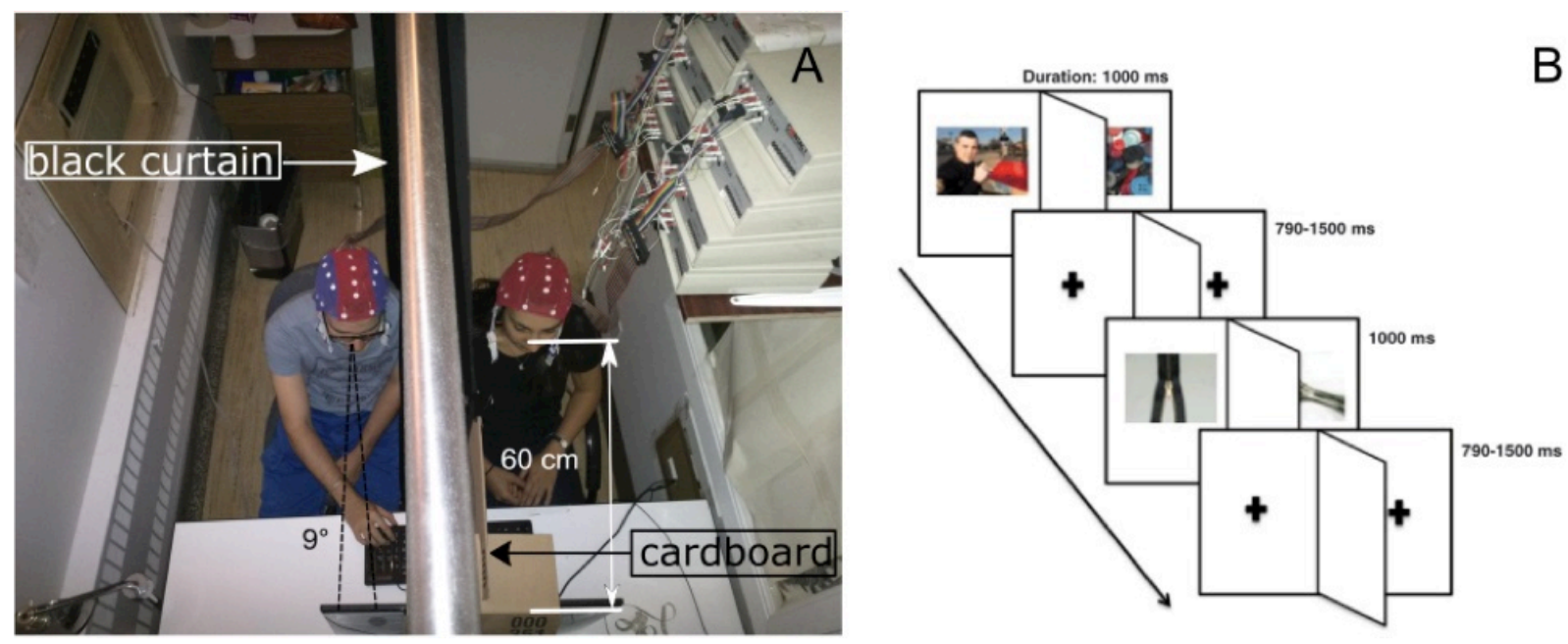

Figure 2. Experimental setup and stimuli presentation.

As in Bouten et al. (2015), during each of the four blocks of stimuli, each of the 70 double picture was displayed for a $1000 \mathrm{~ms}$ duration (Fig. 2B), immediately followed by a black cross, which duration was randomized from 790 to $1500 \mathrm{~ms}$ to prevent the development of a contingent negative variation (Tecce, 1972). At the beginning of each block, a sentence indicated the task and whether pictures were the same for both partners or not. This sentence could be true or false. The two blocks of the "believe same" condition started with the sentence "Try to remember the next 70 images, your partner will be seeing the same images". The two blocks of the "expected different" condition started with the sentence "Try to remember the next 70 images, your partner will be seeing different images". The "consistency" conditions included the two blocks where expectation and reality were consistent (i.e., identical stimuli expected to be the same and different stimuli expected to be different). The "inconsistency" conditions included the two blocks where 
expectation and reality were inconsistent (i.e., identical stimuli expected to be different and different stimuli expected to be the same).

\section{Data acquisition}

For each participant of each pair, the electroencephalogram (EEG) was recorded from 28 tin electrodes of a cap from Electro-Cap International. They were located following the modified expanded 10-20 system (Electrode position nomenclature committee, 1991). The right ear lobe was used as the reference and the ground was placed $2 \mathrm{~cm}$ ahead of Fz. Electrodes were grouped into three subsets: a sagittal (Fz, Fcz, Cz and Pz), a parasagittal (Fp1/2, F3/4, Fc3/4, C3/4, Cp3/4, P3/4, and $\mathrm{O} 1 / 2)$ and a lateral subset (F7/8, Ft7/8, T3/4, Tp7/8 and T5/6). The cap of each of the two partners was connected to one of the two separate sets of amplifiers, both having a 10,000 gain. Their high- and low-pass filters had their half-amplitude cut-offs adjusted at $0.01 \mathrm{~Hz}$ and $100 \mathrm{~Hz}$, respectively. A $60 \mathrm{~Hz}$ electronic notch filter was also used. EEG signals were digitized at a $512 \mathrm{~Hz}$ sampling rate and stored in a single file of 56 channels $(28 \times 2)$.

\section{Data processing and measures}

Baselines were set by computing the mean voltage in the -200 to $0 \mathrm{~ms}$ time window for each electrode and by subtracting this mean value to each point of the -200 to $1200 \mathrm{~ms}$ EEG epoch. Trials contaminated by artifacts were removed offline if they met rejection criteria, that is, if analog-to-digital clipping exceeded a $100 \mathrm{~ms}$ duration and/or if amplitude exceeded $+/-100 \mu \mathrm{V}$. To obtain eight ERPs, one for each of the 4 conditions and for each of the two subjects, the remaining EEG epochs corresponding to the trials of each condition were averaged in the 1400 ms window beginning $200 \mathrm{~ms}$ before the onset of the stimulus and ending $1200 \mathrm{~ms}$ after the onset. Then, the averaged files of 56 channels were divided into two files of 28 channels containing the ERPs of a 
single subject. For one of the subjects of each pair, the ERP obtained for $\mathrm{Cz}$ had to be recomputed using a linear combination of the two nearest electrodes (i.e., $\mathrm{Cz}=(2 \mathrm{FCz}+\mathrm{Pz}) / 3)$ due to a defective amplifier channel. As in Bouten et al. (2015), mean voltages were then measured at all electrodes, for all conditions and all subjects in the N400 time window (350-550 ms after stimulus onset) and in the P600 time window (650-950 ms after the onset).

\section{Statistical analyses}

For each subset of electrodes: sagittal, parasagittal and lateral, the measures were analyzed through a repeated-measures ANOVA using a multivariate approach run with the version 21 of the IBM SPSS software. A two-factor analysis scheme was used, including consistency (stimuli-realsameness being consistent versus inconsistent with the expectation of that sameness) and electrodes as within-subject factors. Each time, for the parasagittal and lateral subsets, the hemiscalp factor (right vs. left) was added. There was no between-subject factor. The Greenhouse and Geisser's (1959) adjustment of degrees of freedom was used to palliate to the heterogeneity of variances across electrodes, in which case the original $\mathrm{F}$ values and degrees of freedom are given together with the corrected $\mathrm{p}$ values.

\section{Results}

Figure 3 shows that the ERPs of the inconsistent conditions were again more positive than those of the consistent conditions. As displayed on Table 1, these differences were significant in the N400 time window for the sagittal and parasagittal electrode subsets. N400s were thus smaller when the participants' expectation about the sameness of the stimuli was inconsistent with the actual sameness. This was observed despite the fact that they had no means to verify their 
expectation since they could not see what their partner was seeing. The debriefing session revealed that most subjects did not feel deceive at any point $(96.9 \%, \mathrm{n}=32)$, and thus did not suspect any falsity in the statements aimed at manipulating sameness expectation. A consistency $\mathrm{x}$ electrodes interaction was also observed in the P600 time window for the sagittal electrodes (Table 1). P600s were larger when the participants' expectation about the sameness of the stimuli was inconsistent with the actual sameness.
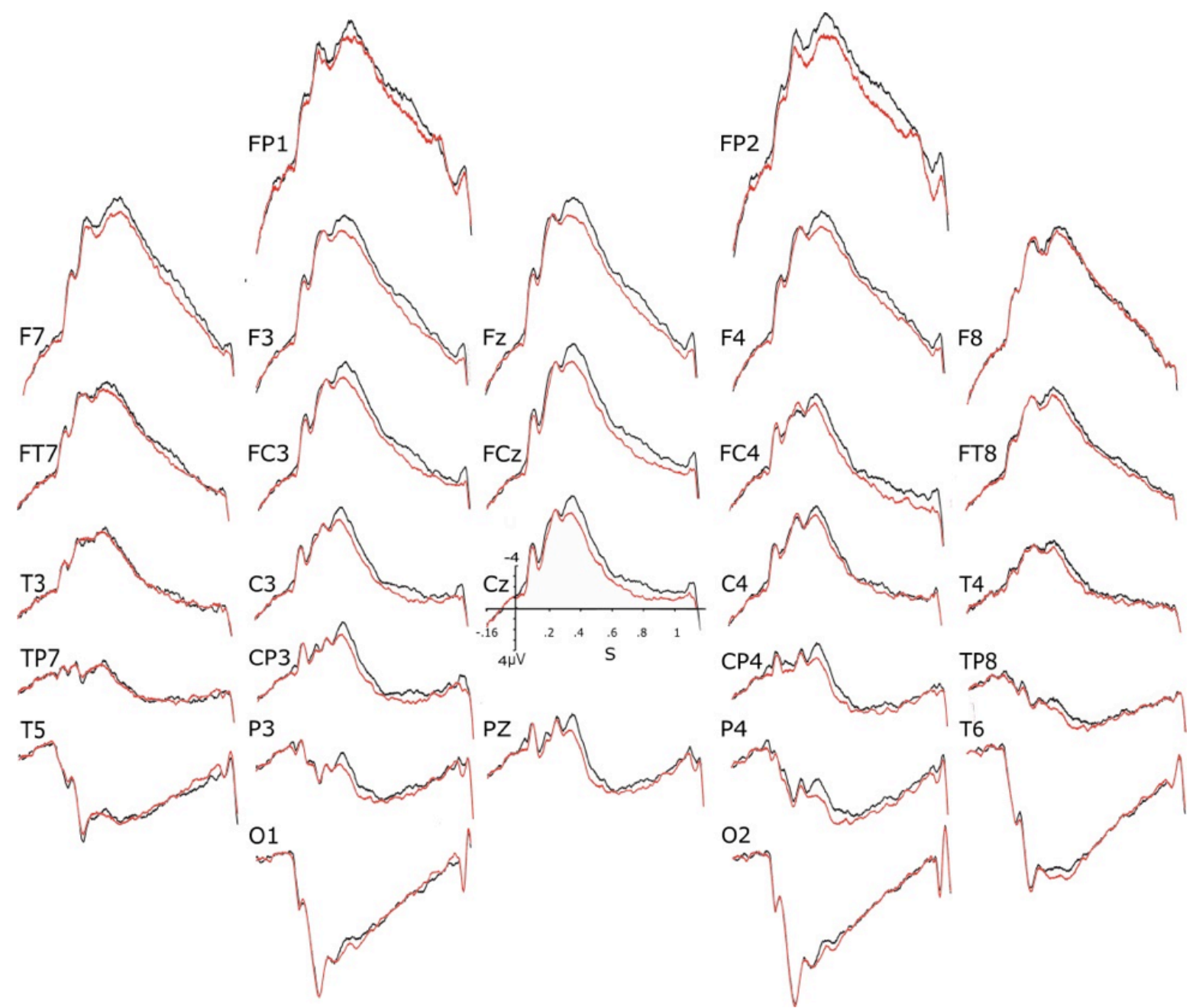

Figure 3. Grand average ERPs of the 32 closely-related participants of the first experiment. As in Fig. 1, the red lines correspond to the two gathered blocks where the reality and the expectation are inconsistent and the black lines correspond to the two gathered blocks where the reality and the expectation are consistent. 


\begin{tabular}{|c|c|c|c|c|}
\hline Electrodes subset & Within-subject factor & $\boldsymbol{F}$ & $D f$ & $\boldsymbol{P}$ \\
\hline \multirow{2}{*}{ 350-550 ms Sagittal } & consistency & 5 & 1,31 & $\mathbf{0 , 0 3}$ \\
\hline & consistency x electrodes & 4,05 & 3,93 & $\mathbf{0 , 0 2}$ \\
\hline \multirow{3}{*}{ Parasagittal } & consistency & 4,84 & 1,31 & 0,04 \\
\hline & & & & \\
\hline & consistency x electrodes & 0,34 & 6,186 & 0,73 \\
\hline \multirow[b]{2}{*}{ Lateral } & consistency & 2,52 & 1,31 & 0,12 \\
\hline & consistency x electrodes & 0,36 & 4,124 & 0,65 \\
\hline \multirow{2}{*}{$650-950 \mathrm{~ms}$ Sagittal } & consistency & 1,33 & 1,31 & 0,26 \\
\hline & consistency $\mathrm{x}$ electrodes & 5,05 & 3,93 & 0,006 \\
\hline \multirow{3}{*}{ Parasagittal } & consistency & 1,49 & 1,31 & 0,23 \\
\hline & & & & \\
\hline & consistency $\mathrm{x}$ electrodes & 1,24 & 6,186 & 0,30 \\
\hline \multirow{2}{*}{ Lateral } & consistency & 0,24 & 1,31 & 0,63 \\
\hline & consistency $\mathrm{x}$ electrodes & 1,28 & 4,124 & 0,29 \\
\hline
\end{tabular}

Table 1. Results of the ANOVAs run with the mean voltages of the ERPs of the 32 closely-related participants of the first experiment measured in the $350-550 \mathrm{~ms}$ and the $650-950 \mathrm{~ms}$ time window. 


\section{Comparison between Bouten et al.'s (2015) study and experiment 1: effect of visual isolation of each subject from his/her partner.}

As the only difference between Bouten et al.'s (2015) study and ours is the presence of the curtain to visually isolate the participants, the results of the two studies were compared. ERPs were analyzed as before adding a between-subjects factor: the presence or absence of the curtain between the two subjects of each pair. As presented in the Table 3, below, there was no significant effect of this visual isolation. Together with the effect on the N400 observed with the closely related people separated with a curtain, this permits to exclude the possibility that the results of the initial study were due to the integration of peripheral visual cues from the partner.

\begin{tabular}{ccccc} 
Electrodes subset & Between-subject factor & $\boldsymbol{F}$ & $\boldsymbol{D f}$ & $\boldsymbol{P}$ \\
\cline { 2 - 5 } $350-550$ ms Sagittal & curtain & 0,04 & 1,62 & 0,85 \\
\hline Parasagittal & curtain & 1,82 & 1,62 & 0,18 \\
\hline Lateral & curtain & 0,39 & 1,62 & 0,54 \\
\hline $650-950$ ms Sagittal & curtain & 0,60 & 1,62 & 0,44 \\
\hline Parasagittal & curtain & 2,19 & 1,62 & 0,14 \\
\hline Lateral & curtain & 0,73 & 1,62 & 0,40
\end{tabular}

Table 2. Absence of curtain effect. Results of the ANOVAs run on mean voltages of ERPs in the N400 and P600 time windows for the two groups of closely-related participants: the group of the first experiment of the present study where the subjects were separated by a curtain $(n=32)$ and the group of Bouten et al. (2015) where they were not separated by a curtain $(n=32)$. 
The 64 participants of the two studies were then grouped (see Figure 4, below) and analyzed. As presented in the upper panels of Table 3 below, the repeated measures ANOVAs show that consistency differences were again significant in the N400 and in the P600 time window for the sagittal, parasagittal and lateral electrodes subsets.

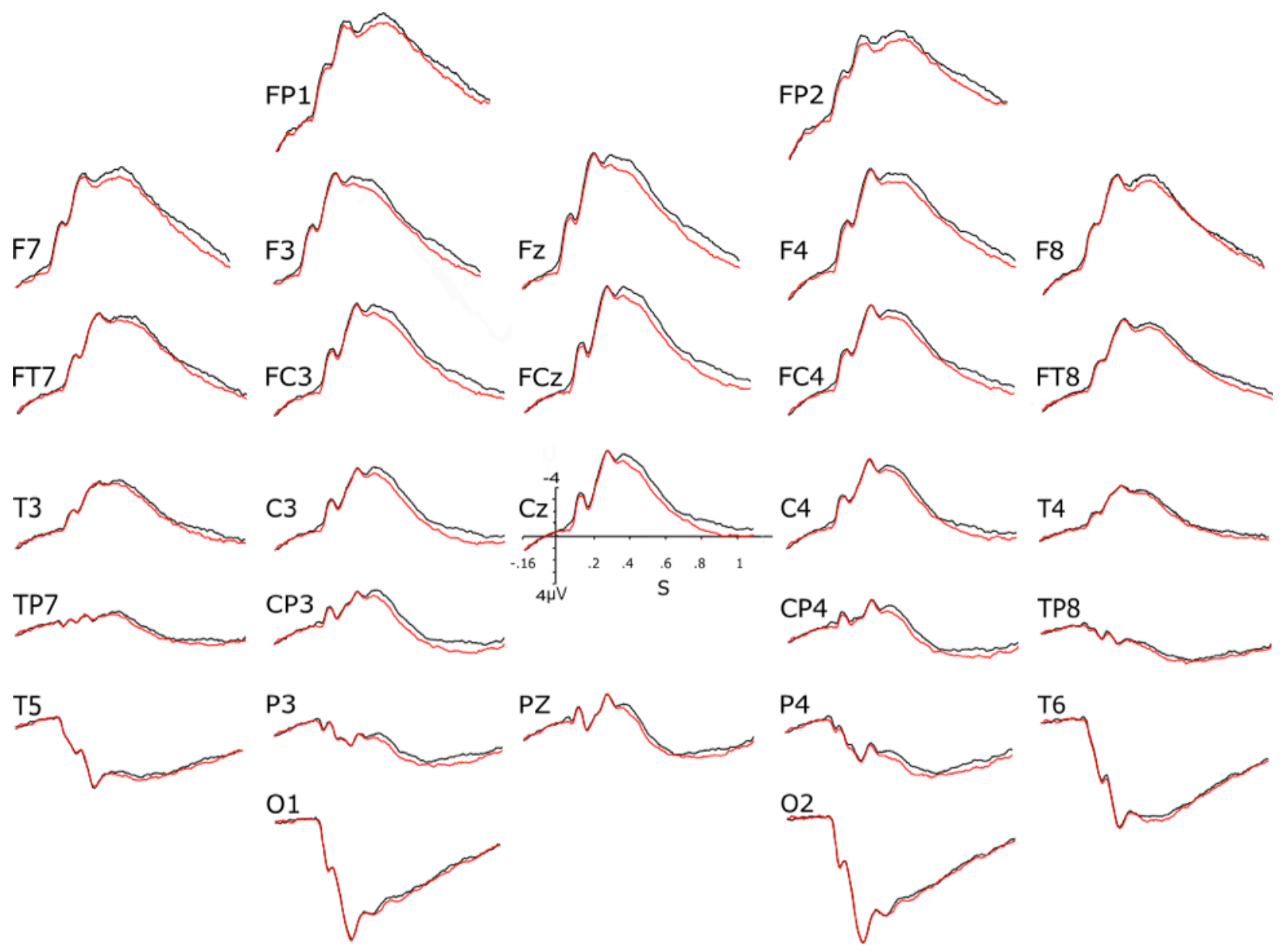

Figure 4. Consistency effect of the 2 studies grouped. Grand $(n=64)$ averages of the ERPs of the 32 closely-related participants of the first experiment of the present study separated by a curtain together with the 32 closely-related participants without curtain of Bouten et al. (2015) The red line corresponds to the two gathered blocks where the reality and the expectation are inconsistent and 
the black line corresponds to the two gathered blocks where the reality and the expectation are consistent.

\begin{tabular}{|c|c|c|c|c|}
\hline Electrode subset & Within-64 subjects factors & $\boldsymbol{F}$ & $D f$ & $\boldsymbol{P}$ \\
\hline \multirow{4}{*}{ 350- 550 ms Sagittal } & $\underline{\text { consistency }}$ & 8,59 & 1,62 & 0,005 \\
\hline & consistency $\mathrm{x}$ electrodes & 2,90 & 3,186 & 0,07 \\
\hline & consistency $\mathrm{x}$ electrodes $\mathrm{x}$ curtain & 1,07 & 3,186 & 0,34 \\
\hline & consistency & 7,16 & 1,62 & $\mathbf{0 , 0 1}$ \\
\hline \multirow[t]{3}{*}{ Parasagittal } & consistency $\mathrm{x}$ electrodes & 0,60 & 6,372 & 0,57 \\
\hline & consistency $\mathrm{x}$ electrodes $\mathrm{x}$ curtain & 0,13 & 6,372 & 0,90 \\
\hline & consistency & 4,98 & 1,62 & $\mathbf{0 , 0 2 9}$ \\
\hline \multirow[t]{3}{*}{ Lateral } & consistency $\mathrm{x}$ electrodes & 1,16 & 4,248 & 0,31 \\
\hline & consistency $\mathrm{x}$ electrodes $\mathrm{x}$ curtain & 0,13 & 4,248 & 0,84 \\
\hline & consistency & 7,92 & 1,62 & $\mathbf{0 , 0 0 7}$ \\
\hline \multirow[t]{3}{*}{$650-950$ ms Sagittal } & consistency $\mathrm{x}$ electrodes & 4,95 & 3,186 & 0,009 \\
\hline & consistency $\mathrm{x}$ electrodes $\mathrm{x}$ curtain & 0,97 & 3,186 & 0,38 \\
\hline & consistency & 5,94 & 1,62 & 0,018 \\
\hline \multirow[t]{3}{*}{ Parasagittal } & consistency $\mathrm{x}$ electrodes & 0,93 & 6,372 & 0,40 \\
\hline & consistency $\mathrm{x}$ electrodes $\mathrm{x}$ curtain & 1,66 & 6,372 & 0,19 \\
\hline & consistency & 2,64 & 1,62 & 0,11 \\
\hline \multirow[t]{2}{*}{ Lateral } & consistency $\mathrm{x}$ electrodes & 1,20 & 4,248 & 0,30 \\
\hline & consistency $\mathrm{x}$ electrodes $\mathrm{x}$ curtain & 0,62 & 4,248 & 0,53 \\
\hline
\end{tabular}

Table 3. Results of the ANOVAs run on the mean voltages of the ERPs in the N400 and P600 time windows (450-550 \& 650-950ms post-onset) for the 64 subjects of the two studies with consistency (actual sameness of the stimulus presented to the partner and expectation of the subject in that 
sameness are consistent versus inconsistent with each other) and electrodes as within-subject factors.

\section{Discussion}

This fruitful attempt at replicating the difference observed in the preliminary study of Bouten et al. (2015) showed that when partners could not see each other because of the curtain separating them, significant differences between the two consistency conditions remained. As subjects could not see the stimulus of their partner, the results support the hypothesis that the brain of a subject emits, in response to the presentation of a stimulus, a physical phenomenon that can be detected by its impact on the activity of the brain of a closely-related person.

Given the importance that such results would have, it is crucial to ensure the absence of any bias and of any alternate account. The only way the present ERP results can be explained without the hypothesized emissions is by a classical detection of the inconsistencies between the expectation-inducing-statement and the stimuli presented to the partner. Given that no deception was reported by subjects at the debriefing session, such a detection would have had to be unconscious. At first sight, this does not seem impossible, even with the curtain. As mentioned, a significant number of pictures were emotionally-loaded. Thus, they could also have triggered breathing variations that could have been preconsciously detected through the curtain by the partner's ear. Such variations could have been found consistent or inconsistent with the emotions triggered by the stimulus presented to this partner. This could have then been used by the system to discover that the statement was deceiving for the trial at stake. Then, from the few trials were such unconscious discoveries would have been made, a covert extrapolation to all or, at least to the 
majority of, the trials of the block could have happened. However, one has to admit that meeting all these criteria is unlikely and that such a generalization of a covert finding looks quite implausible. Moreover, the fact that P600s were larger in the inconsistent- than in the consistent-conditions would also be a bit puzzling within that perspective. The amplitude of the P600, a component of the P3b family (Kok, 2001), sometimes termed late posterior positivity (LPP), correlates positively with the amount of information consciously extracted from the trial (e.g., Kutas et al., 1977; Donchin \& Coles, 1988; Sergent et al., 2005). Accordingly, stimuli presented in the inconsistent blockconditions led to the consciousness of a greater amount of information than the stimuli of the consistent conditions. There seems to be no reason why an unconscious detection of deception by the system would have led to such greater conscious amounts. In contrast, the N400 is known to index preconscious processing (Sergent et al., 2005; Van Gaal et al., 2014; Vogel et al., 1998). Thus, one could have argued that the earlier part of the ERP effect of consistency was due to the covert detection. However, the direction of the effect would again be surprising. Given that the amplitude of the N400 is greater with stimuli that are incongruent with predictions (for a review, see Kutas \& Federmeier, 2011), one would have expected greater N400s for inconsistent than for consistent block-conditions. The fact that the contrary was observed goes also against the alternate account. Thus, the possibility that a classical but covert detection of inconsistency made at a few emotionally loaded trials would have impacted the processing of the majority of the trials of the block is not only unlikely, it also fails to make sense of the electrophysiological data observed.

On the contrary, the effect of consistency found here on ERPs supports the emission theory. As hypothesized, the percepts produced by the brain of a participant would be emitted and would impact his/her brain and the brain of his/her partner. Such a view allows us to avoid the mind-body problem, which is created by the idea that the brain, which is physical, would be able to produce a 
mind of a non-physical nature. This view may also provide us with a way to understand how percepts can be similar across individuals having brains with different learning histories.

\section{Experiment 2: Testing whether consistency effects on ERPs can be obtained with strangers}

\section{Introduction}

The ERP effects obtained in Bouten et al.'s (2015) study and those of the present study were obtained with pairs of participants who were closely related to each other, as it was assumed that being in such a relation could only increase the odds of a sensitivity to the partner. The second experiment of the present study thus aims at testing whether such ERP effects would be found with subjects who did not know each other before the experiment.

\section{Methods}

The same method as Experiment 1 was used except that the thirty-four right-handed English speaking participants recruited $(10 \mathrm{M}, 24 \mathrm{~F}$, mean age $=22.05$ years, $\mathrm{SD}=2.46)$ did not know each other. The same inclusion/exclusion criteria as Experiment 1 were used to recruit them. The same procedure as in Bouten et al. (2015) was applied, that is, only a cardboard was masking the stimulus presented to the partner (Figure 8, A). No curtain was used to separate them, as if the absence of a consistency effect on ERPs in those conditions would a) further strengthen the idea that the results of Bouten et al. (2015) were not due to visual communications between the two participants of each pair and b) permit to make sure that the ERP effect does not occur with strangers even if the feeling of the presence of the other is increased by being able to see him/her in the very periphery of one's visual field. All else, that is, consent, stimuli, procedure, data acquisition, statistical analyses, were as in Experiment 1. Note that, again, for one of the subjects of pairs 1, 4, 7 and 15, the ERP 
obtained for $\mathrm{Cz}$ had to be recomputed using a linear combination of the two nearest electrodes (i.e., $\mathrm{Cz}=(2 \mathrm{FCz}+\mathrm{Pz}) / 3)$ due to the defective amplifier. The EEG also appeared problematic for the FC4 channel for one of the subjects of each pair. It was also recalculated using a linear combination of the two nearest electrodes (i.e., $(\mathrm{F} 4+\mathrm{C} 4) / 2)$.

\section{Results}

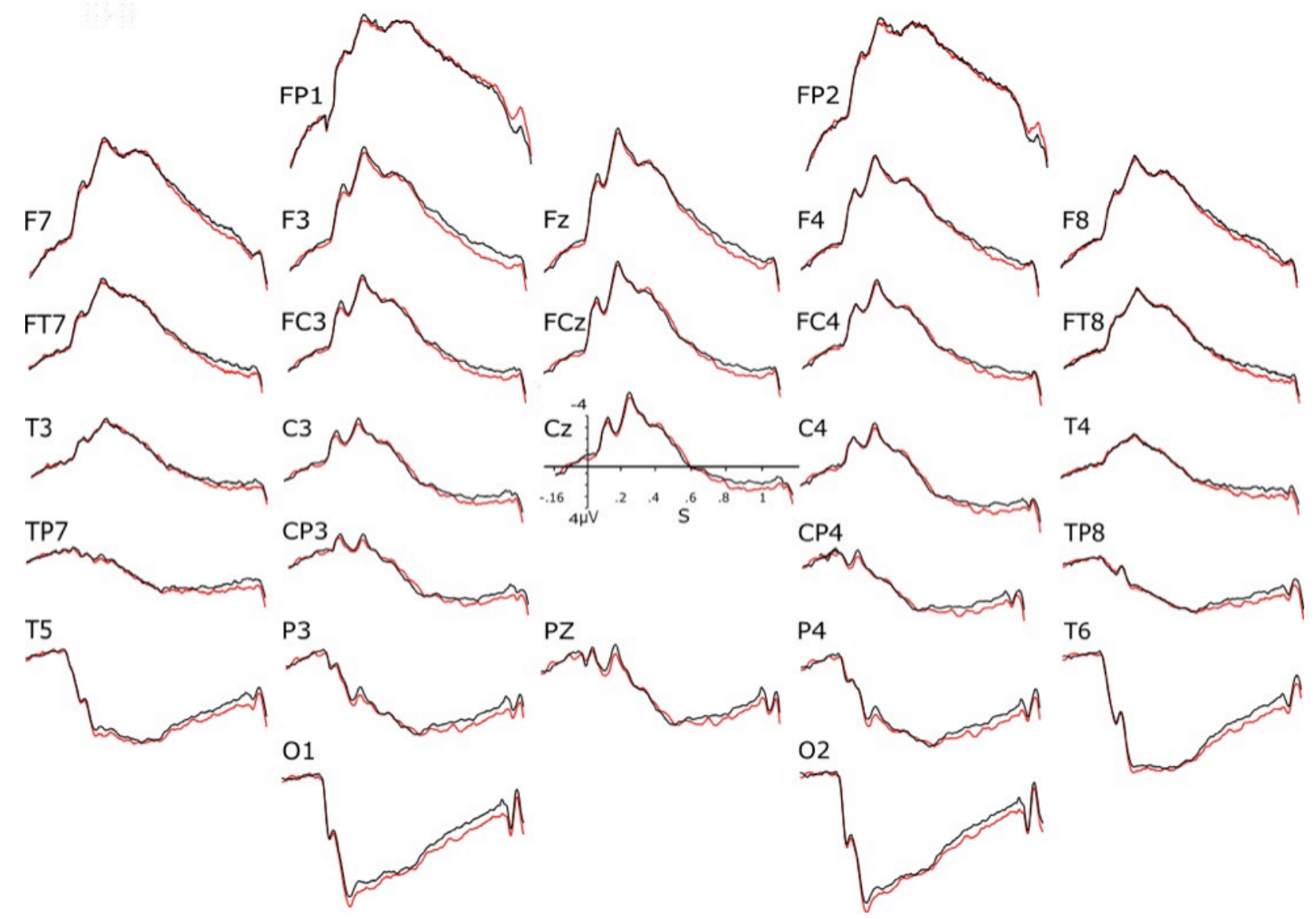

Figure 5. Grand averages of the ERPs of the 34 strangers of the second experiment. The black lines correspond to the two gathered blocks where the reality and the expectation were consistent, the red lines correspond to the two gathered blocks where the reality and the expectation were inconsistent. 
The visual inspection of these ERPs (Fig. 5) reveals late differences in the direction of those found in Bouten al.'s (2015) study and in the first experiment of the present study. Nevertheless, they look of smaller size and the repeated-measures ANOVAs performed on the mean voltages of the N400 and P600 time windows for the 34 strangers showed no significant effect of consistency as well as no interaction of this factor with electrode for any of the subsets. These findings suggest that the consistency effect of Bouten et al.'s (2015) study does not occur, or is too small, within dyads of people who do not know each other.

\section{Comparison between partners and strangers: effect of the prior relationship}

To test whether the consistency effect depends on the relationship between participants, we compared the one obtained in the 64 subjects ( 32 with the curtain and 32 without the curtain) in a close relationship to that obtained in the 34 strangers. As presented in the upper panel of Table 4 , these repeated-measures ANOVAs revealed a triple interaction between consistency, electrodes and relationship for the sagittal electrode subset in the N400 time window. A post-hoc revealed that the consistency effect was significantly smaller for strangers than for partners at $\mathrm{Fz}(F(1,96)=5,16$, $P=0,025)$ and $\mathrm{FCz}(F(1,96)=6,83, P=0,01)$ in this time window. 
Running head: Physicality of mind

\begin{tabular}{|c|c|c|c|c|}
\hline Electrodes subset & Factors & $\boldsymbol{F}$ & $D f$ & $\boldsymbol{P}$ \\
\hline \multirow[b]{2}{*}{ 350-550 ms Sagittal } & consistency x relationship & 2,89 & 1,96 & 0,09 \\
\hline & $\begin{array}{c}\text { consistency } \mathrm{x} \text { electrodes } \mathrm{x} \\
\text { relationship }\end{array}$ & 2,90 & 3,288 & $\mathbf{0 , 0 3}$ \\
\hline \multirow{3}{*}{ Parasagittal } & consistency x relationship & 3,40 & 1,96 & 0,07 \\
\hline & $\begin{array}{c}\text { consistency } \mathrm{x} \text { electrodes } \mathrm{x} \\
\text { relationship }\end{array}$ & 1,74 & 6,576 & 0,17 \\
\hline & consistency $\mathrm{x}$ relationship & 2,29 & 1,96 & 0,13 \\
\hline Lateral & $\begin{array}{c}\text { consistency } \mathrm{x} \text { electrodes } \mathrm{x} \\
\text { relationship }\end{array}$ & 0,71 & 4,384 & 0,47 \\
\hline \multirow[b]{2}{*}{$650-950 \mathrm{~ms}$ Sagittal } & consistency x relationship & 0,50 & 1,96 & 0,48 \\
\hline & $\begin{array}{c}\text { consistency } \mathrm{x} \text { electrodes } \mathrm{x} \\
\text { relationship }\end{array}$ & 0,97 & 3,288 & 0,38 \\
\hline \multirow{3}{*}{ Parasagittal } & consistency $\mathrm{x}$ relationship & 0,21 & 1,96 & 0,65 \\
\hline & $\begin{array}{c}\text { consistency } \mathrm{x} \text { electrodes } \mathrm{x} \\
\text { relationship }\end{array}$ & 0,84 & 6,576 & 0,45 \\
\hline & consistency x relationship & 0,001 & 1,96 & 0,98 \\
\hline Lateral & $\begin{array}{c}\text { consistency } \mathrm{x} \text { electrodes } \mathrm{x} \\
\text { relationship }\end{array}$ & 0,61 & 4,384 & 0,54 \\
\hline
\end{tabular}

Table 4. Results of the ANOVAs run on the mean voltages of the ERPs in the N400 (350-550 ms) and in the P600 time window (650-950 ms) for closely-related participants $(n=64)$ and for strangers $(n=34)$. 


\section{Conclusion}

In Bouten et al.'s (2015) experiment, the ERP differences observed could have been due the visual peripheral cues (body or head movements) that could have covertly indicated to one partner the valence of the image seen by the other. A curtain was thus installed to visually isolate the subjects. The differences between the consistent and inconsistent conditions remained, which suggests that the effect could not be explained by the partner's body language. This replication of the effect can thus be used to support the theory of an emission of percepts. The same procedure was done with strangers and the consistency effect was not found, suggesting that prior interactions are necessary for the effect to occur.

Although the sensitivity to percepts of others appears theoretically necessary to produce qualia that are similar across individuals, as mentioned in Bouten et al.'s (2015) study, nothing in their data or in the present ones indicates that the sensitivity is used for this purpose. Although constantly assumed in our everyday life interactions with others, how this similarity can exist despite the large differences existing between individual brains have, to the best of our knowledge, never been searched for. Further investigations would thus be interesting. On the other hand, studying the nature of the physics involved in (conscious) percepts and the means by which brains are sensitive to them should be the focus of interdisciplinary approaches. 


\section{References}

Ancient Visions. (n.d.). http://nivea.psycho.univ-paris5.fr/FeelingSupplements/AncientVisions.htm

Bodart, O., Gosseries, O., Wannez, S., Thibaut, A., Annen, J., Boly, M., ... Laureys, S. (2017).

Measures of metabolism and complexity in the brain of patients with disorders of consciousness.

NeuroImage: Clinical, 14, 354-362. https://doi.org/10.1016/j.nicl.2017.02.002

Bouten, S., Pantecouteau, H., \& Debruille, J. B. (2015). Finding indexes of spontaneous brain-to-

brain communications when looking for a cause of the similarity of qualia assumed across

individuals. F1000Research. https://doi.org/10.12688/f1000research.5977.2

Chalmers, D. (2014). How do you explain consciousness?. Ted Talks.

Dehaene, S. (2001). Towards a cognitive neuroscience of consciousness: Basic evidence and a workspace framework. Cognition, 79(1-2), 1-37. doi:10.1016/s0010-0277(00)00123-2

Dehaene, S., Charles, L., King, J.-R., \& Marti, S. (2014). Toward a computational theory of conscious processing. Current Opinion in Neurobiology, 25, 76-84.

https://doi.org/10.1016/j.conb.2013.12.005

Donchin, E., \& Coles, M. G. (1988). Is the P300 component a manifestation of context updating. Behavioral and brain sciences, 11(3), 357-427. 
Electrode position nomenclature committee. (1991). American Electroencephalographic Society guidelines for standard electrode position nomenclature. Journal of Clinical Neurophysiology, 8(2), 200-202

Greenhouse, S. W., \& Geisser, S. (1959). On methods in the analysis of profile data. Psychometrika, 24(2), 95-112. doi:10.1007/bf02289823

Huang, Z., Zhang, J., Wu, J., Qin, P., Wu, X., Wang, Z., ... Northoff, G. (2016). Decoupled temporal variability and signal synchronization of spontaneous brain activity in loss of consciousness: An fMRI study in anesthesia. NeuroImage, 124, Part A, 693-703. https://doi.org/10.1016/j.neuroimage.2015.08.062

Kok, A. (2001). On the utility of P3 amplitude as a measure of processing capacity. Psychophysiology, 38, 557-77.

Kutas, M., McCarthy, G., \& Donchin, E. (1977). Augmenting mental chronometry: the P300 as a measure of stimulus evaluation time. Science, 197(4305), 792-795.

Kutas, M. \& Federmeier, K. D. (2011) Thirty years and counting: Finding meaning in the N400 component of the event related brain potential (ERP). Annu. Rev. Psychol. 62, 621-647.

Lang, P.J., Bradley, M.M., Cuthbert, B.N., and others (1997). Technical Manual and Affective Ratings. NIMH Center for the Study of Emotion and Attention. 
Lesser, R. P. (1986). American Electroencephalographic society guidelines in EEG, 1-7 (revised 1985). Journal of Clinical Neurophysiology, 3(2), 131-132. doi:10.1097/00004691-19860400000003

Sergent, C., Baillet, S., \& Dehaene, S. (2005). Timing of the brain events underlying access to consciousness during the attentional blink. Nature neuroscience, 8(10), 1391-1400.

Silverstein, B. H., Snodgrass, M., Shevrin, H., \& Kushwaha, R. (2015). P3b, consciousness, and complex unconscious processing. Cortex, 73, 216-227. https://doi.org/10.1016/j.cortex.2015.09.004

Tecce, J. J. (1972). Contingent negative variation (CNV) and psychological processes in man. Psychological Bulletin, 77(2), 73-108. doi:10.1037/h0032177

Tononi, G., \& Edelman, G. M. (1998). Consciousness and complexity. Science (New York, N.Y.), $282(5395), 1846-1851$.

Tononi, G., Boly, M., Massimini, M., \& Koch, C. (2016). Integrated information theory: from consciousness to its physical substrate. Nature Reviews Neuroscience, 17(7), 450-461. https://doi.org/10.1038/nrn.2016.44

Van Gaal, S., Naccache, L., Meuwese, J. D. I., van Loon, A. M., Leighton, A. H., Cohen, L., \& Dehaene, S. (2014). Can the meaning of multiple words be integrated unconsciously? Philosophical Transactions of the Royal Society B: Biological Sciences, 369(1641), doi:10.1098/rstb.2013.0212 
Running head: Physicality of mind

Vogel, E. K., Luck, S. J. \& Shapiro, K. L. (1998) Electrophysiological evidence for a

postperceptual locus of suppression during the attentional blink. J. Exp. Psychol.-Hum. Percept.

Perform. 24(6), 1656-74. 
Running head: Physicality of mind

Acknowledgments: This study has been supported by the grant 194517-03 from NSERC allocated to the corresponding author. We thank the subjects for their participation.

Data availability: all data, including raw EEG files (in a MatLab format) will be sent on request to bruno.debruille@mcgill.ca 